\title{
An Inequality for Closed Manifolds with Timelike Immersion and Negative Gauss Curvature
}

\author{
Serpil Karagoz \\ Faculty of Art and Sciences, Mathematics Department, Abant Izzet Baysal University, Bolu, Turkey.
}

*Corresponding Author: Serpil Karagoz, Faculty of Art and Sciences, Mathematics Department, Abant Izzet Baysal University, Bolu, Turkey.

Abstract: In this paper an inequality for closed manifolds with timelike immersion and negative Gauss curvature is derived. It is computed by means of the mean curvature $H$ and Gauss curvature $G$ of timelike immersed manifold.

$$
\text { - } \int_{V} H^{2} d V \geq \int_{V} \sqrt{\left|-3 G^{2}-2\right|} d V \text {. }
$$

Keywords: Total Absolute Curvatue, Lorentz Space, Timelike Immersion, Mean Curvature, Gauss Curvature

\section{INTRODUCTION}

Let $M^{2}$ be an oriented closed surface with a timelike immersion : $M^{2} \rightarrow L^{4}$. Let $F\left(M^{2}\right)$ and $F\left(L^{4}\right)$ be the bundles of orthonormal frames $M^{2}$ and $L^{4}$ respectively. Let $\mathrm{B}$ be the set of elements $b=\left(p, l_{1}, l_{2}, l_{3}, l_{4}\right)$ such that $\left(p, l_{1}, l_{2}\right) \in F\left(M^{2}\right)$ and $b=\left(x(p), l_{1}, l_{2}, l_{3}, l_{4}\right) \in F\left(L^{4}\right)$ whose orientation is coherent with the one of $L^{4}$, identifying $l_{i}$ with $d x\left(l_{i}\right), i=1,2$ where $l_{i}$ are unit vectors and $l_{2}$ is a timelike vector.

Define $\widetilde{x}: B \rightarrow F\left(L^{4}\right)$ naturally by $b \rightarrow\left(x(p), l_{1}, l_{2}, l_{3}, l_{4}\right)$. The structure equations of $L^{4}$ are given by

$$
\begin{gathered}
d x=\sum \widetilde{w}_{A} l_{A} \quad d l_{A}=\sum \widetilde{w}_{A B} l_{B} \quad \widetilde{w}_{A B}+\widetilde{w}_{B A}=0 \\
d \widetilde{w}_{A}=\sum \widetilde{w}_{B} \Lambda \widetilde{w}_{B A} \quad d \widetilde{w}_{A B}=\sum \widetilde{w}_{A C} \Lambda \widetilde{w}_{C B} \quad A, B, C=1,2,3,4
\end{gathered}
$$

where $\widetilde{w}_{A}, \widetilde{w}_{A B}$ are differential 1- forms on $F\left(L^{4}\right)$.

Let $w_{A}, w_{A B}$ be induced 1 - forms on $\mathrm{B}$ from $\widetilde{w}_{A}, \widetilde{w}_{A B}$ by the mapping $\widetilde{x}$. Then we have

$$
\begin{gathered}
w_{3}=w_{4}=0 \\
w_{i 3}=A_{3 i 1} w_{1}+A_{3 i 2} w_{2} \\
w_{i 4}=A_{4 j 1} w_{1}+A_{4 j 2} w_{2} ; i, j=1,2
\end{gathered}
$$

Let $\left(p, l_{1}, l_{2}, \tilde{l}_{3}, \tilde{l}_{4}\right)$ be a local cross-section of $B \rightarrow F\left(M^{2}\right)$. The restriction of $A_{\text {rij }}$ onto the image of local cross-section is denoted by $\bar{A}_{\text {rij }}$ where $=3,4$.

We can compute second fundamental form as

$$
I I(d p, d p)=<S(d p), d p>
$$

where $S$ is the shape operator of the immersion

$$
\begin{gathered}
I I(d p, d p)=w_{1}^{2}<S\left(l_{1}\right), l_{1}>+2 w_{1} w_{2}<S\left(l_{1}\right), l_{2}>+w_{2}^{2}<S\left(l_{2}\right), l_{2}> \\
S\left(l_{1}\right)=D_{11} l_{4}=A_{411} l_{1}-A_{421} l_{2} \\
S\left(l_{2}\right)=D_{12} l_{4}=A_{412} l_{1}-A_{422} l_{2}
\end{gathered}
$$




$$
\begin{gathered}
S=\left(\begin{array}{ll}
A_{411} & -A_{421} \\
A_{412} & -A_{422}
\end{array}\right) \\
I I(d p, d p)=A_{411} w_{1}^{2}+2 A_{412} w_{1} w_{2}+A_{422} w_{2}^{2}
\end{gathered}
$$

is the second fundamental form.

Theorem 1: Let $M^{2}$ be a 2-dimensional oriented closed manifold with a timelike immersion : $M^{2} \rightarrow$ $L^{4}$. If $\left(A_{4 i j}\right)$ is the shape operator of the timelike immersion then Lipschitz- Killing curvature $K(p, l)$ is given by

$$
K(p, l)=-\lambda_{1}(p) \cos ^{2} \theta-\lambda_{2}(p) \sin ^{2} \theta
$$

where $l$ is the unit normal vector and

$$
\begin{aligned}
& \lambda_{1}(p)=\operatorname{det}\left(\bar{A}_{3 i j}\right) \\
& \lambda_{2}(p)=\operatorname{det}\left(\bar{A}_{4 i j}\right)
\end{aligned}
$$

Proof: Choose $l$ as

$$
\begin{gathered}
l=l_{4}=\cos \theta \tilde{l}_{3}+\sin \theta \tilde{l}_{4} \\
A_{4 i j}=\cos \theta \bar{A}_{3 i j}+\sin \theta \bar{A}_{4 i j} ; i, j=1,2
\end{gathered}
$$

The Lipschitz- Killing curvature $K(p, l)$ is determined by

$K(p, l) \equiv \operatorname{det} A_{4 i j}=\operatorname{det}\left(\begin{array}{ll}\cos \theta \bar{A}_{311}+\sin \theta \bar{A}_{411} & -\cos \theta \bar{A}_{312}-\sin \theta \bar{A}_{412} \\ \cos \theta \bar{A}_{312}+\sin \theta \bar{A}_{412} & -\cos \theta \bar{A}_{322}-\sin \theta \bar{A}_{422}\end{array}\right)$

The determinant is a quadratic form of $\cos \theta$ and $\sin \theta$. It will be derived as

$$
K(p, l)=-\lambda_{1}(p) \cos ^{2} \theta-\lambda_{2}(p) \sin ^{2} \theta
$$

By using an orthonormal frame where

$$
\begin{aligned}
& \lambda_{1}(p)=\operatorname{det}\left(\bar{A}_{3 i j}\right) \\
& \text { and } \\
& \lambda_{2}(p)=\operatorname{det}\left(\bar{A}_{4 i j}\right)
\end{aligned}
$$

$\lambda_{1}(p), \lambda_{2}(p)$ are continuous on $M^{2}$. The Gauss curvature $G(p)$ is given by

$$
G(p)=\lambda_{1}(p)+\lambda_{2}(p)
$$

as in [1].

Theorem 2: Let $M^{2}$ be a 2-dimensional oriented closed manifold with a timelike immersion : $M^{2} \rightarrow$ $L^{4}$. If $G(p)=\lambda_{1}(p)+\lambda_{2}(p)$ is negative Gauss curvature of $M^{2}$ then the total absolute curvature $K^{*}(p)$ at point $p$ is

$$
K^{*}(p)=-\pi G(p)
$$

on $V$ and

$$
K^{*}(p)=(2 \alpha-\pi) G(p)+4 \sqrt{-\lambda_{1} \lambda_{2}}
$$

on $U$ where

$$
\begin{aligned}
& U=\left\{p \in M^{2}, \lambda_{1}(p)>0\right\} \\
& V=\left\{p \in M^{2}, \lambda_{1}(p) \leq 0\right\}
\end{aligned}
$$

Proof: Since $\lambda_{1}$ and $\lambda_{2}$ are both negative on $V$ we have

$K^{*}(p)=\int_{0}^{2 \pi}|K(p, l)| d \theta \quad$ where $K(p, l)$ is the Lipschitz- Killing curvature 


$$
\begin{aligned}
K^{*}(p) & =\int_{0}^{2 \pi}|K(p, l)| d \theta \\
& =\int_{0}^{2 \pi}\left|-\lambda_{1}(p) \cos ^{2} \theta-\lambda_{2}(p) \sin ^{2} \theta\right| d \theta \\
& =\int_{0}^{2 \pi}|-1|\left|\lambda_{1}(p) \cos ^{2} \theta+\lambda_{2}(p) \sin ^{2} \theta\right| d \theta \\
& =\int_{0}^{2 \pi}-\left(\lambda_{1}(p) \cos ^{2} \theta+\lambda_{2}(p) \sin ^{2} \theta\right) d \theta \\
& =-\pi\left(\lambda_{1}(p)+\lambda_{2}(p)\right) \\
& =-\pi G(p)
\end{aligned}
$$

Since $\lambda_{1}$ is positive on $U$ and $G(p) \leq 0$ we have a negative $\lambda_{2}$ such that $\left|\lambda_{2}\right| \geq\left|\lambda_{1}\right|$.

Total absolute curvature on $U$ is

$$
\begin{aligned}
K^{*}(p) & =\int_{0}^{2 \pi}|K(p, l)| d \theta \\
& =\int_{0}^{2 \pi}\left|-\lambda_{1}(p) \cos ^{2} \theta-\lambda_{2}(p) \sin ^{2} \theta\right| d \theta \\
& =\int_{0}^{2 \pi}|-1|\left|\lambda_{1}(p) \cos ^{2} \theta+\lambda_{2}(p) \sin ^{2} \theta\right| d \theta \\
& =\frac{\mathbf{1}}{2} \int_{0}^{2 \pi}\left|\left(\lambda_{1}+\lambda_{2}\right)+\left(\lambda_{1}-\lambda_{2}\right) \cos 2 \theta\right| d \theta \\
& =\frac{1}{2}\left(\lambda_{1}-\lambda_{2}\right) \int_{0}^{2 \pi}\left|\frac{\lambda_{1}+\lambda_{2}}{\lambda_{1}-\lambda_{2}}+\cos 2 \theta\right| d \theta
\end{aligned}
$$

Define an angle $\alpha$ such that

$$
\begin{aligned}
\cos \alpha & =-\frac{\lambda_{1}+\lambda_{2}}{\lambda_{1}-\lambda_{2}} ; \quad 0<\alpha \leq \frac{\pi}{2} \quad \text { so } \quad \sin \alpha=\frac{2 \sqrt{-\lambda_{1} \lambda_{2}}}{\lambda_{1}-\lambda_{2}} \\
K^{*}(p) & =\frac{1}{2}\left(\lambda_{1}-\lambda_{2}\right) \int_{0}^{2 \pi}|\cos 2 \theta-\cos \alpha| d \theta \\
& =\frac{1}{4}\left(\lambda_{1}-\lambda_{2}\right) \int_{0}^{4 \pi}|\cos t-\cos \alpha| d t \\
& =(2 \alpha-\pi) G(p)+4 \sqrt{-\lambda_{1} \lambda_{2}}
\end{aligned}
$$

Theorem 3: Let $M^{2}$ be a 2-dimensional oriented closed manifold with a timelike immersion : $M^{2} \rightarrow$ $L^{4}$. If $G(p)=\lambda_{1}(p)+\lambda_{2}(p)$ is negative Gauss curvature of $M^{2}$ then for the mean curvature $\mathrm{H}$ of $M^{2}$ in $L^{4}$ we have

$$
\int_{V} H^{2} d V \geq \int_{V} \sqrt{\left|-3 G^{2}-2\right|}
$$

Proof: Let for the frame $\left(p, l_{1}, l_{2}, l_{3}, l_{4}\right) ; l_{1}$ and $l_{2}$ be the principal directions with respect to $l_{4}$. Choose $\bar{A}_{\text {rij }}$ as follows

$$
\begin{aligned}
& \bar{A}_{311}=a ; \quad \bar{A}_{312}=\bar{A}_{321}-c ; \bar{A}_{322}=-b \\
& \bar{A}_{411}=d ; \bar{A}_{422}=-e ; \bar{A}_{412}=\bar{A}_{421}=0
\end{aligned}
$$

where a,b,c,d,e are all positive. $\bar{A}_{3 i j}=\left(\begin{array}{cc}a & -c \\ -c & -b\end{array}\right)$ and $\bar{A}_{4 i j}=\left(\begin{array}{cc}d & 0 \\ 0 & -e\end{array}\right)$ then

$$
\begin{gathered}
\lambda_{1}(p)=\operatorname{det}\left(\bar{A}_{3 i j}\right)=-a b-c^{2} \\
\lambda_{2}(p)=\operatorname{det}\left(\bar{A}_{4 i j}\right)=-d e
\end{gathered}
$$

where $\lambda_{2} \leq \lambda_{1} \leq 0$. 
Shape operator is given by $S=\left(\begin{array}{cccc}a & -c & 0 & 0 \\ -c & -b & 0 & 0 \\ 0 & 0 & d & 0 \\ 0 & 0 & 0 & -e\end{array}\right)$. Mean curvature is then

$$
\begin{gathered}
H=\frac{a-b+d-e}{2} \\
H^{2}=\frac{(a-b+d-e)^{2}}{4} \\
4 H^{2}=(a-b)^{2}+(d-c)^{2}+2(a-b)(d-e) \\
=(a-b)^{2}+(d-c)^{2}+2(a d-a e-b d+b e)
\end{gathered}
$$

Since

We have

$$
\begin{gathered}
\bar{A}_{311} \bar{A}_{411}+\bar{A}_{322} \bar{A}_{422}=\bar{A}_{311} \bar{A}_{422}+\bar{A}_{322} \bar{A}_{411}=\bar{A}_{312} \bar{A}_{412}+\bar{A}_{421} \bar{A}_{312} \\
a d-a e-b d+b e=0
\end{gathered}
$$

$$
\begin{gathered}
4 H^{2}=(a-b)^{2}+(d-e)^{2} \\
4 H^{2} \geq 4|a b|+4|d e| \\
4 H^{2} \geq 8 \sqrt{|a b d e|} \\
\lambda_{1} \lambda_{2}=\left(-a b-c^{2}\right)(-d e) \\
=a b d e+d e c^{2} \\
a b d e=\lambda_{1} \lambda_{2}-d e c^{2} \\
\text { abde }=\lambda_{1} \lambda_{2}+\lambda_{2} c^{2}
\end{gathered}
$$

Let $c=1$ we have $a b d e=\lambda_{1} \lambda_{2}+\lambda_{2}$

$$
\begin{gathered}
4 H^{2} \geq 8 \sqrt{\left|\lambda_{1} \lambda_{2}+\lambda_{2}\right|} \\
H^{2} \geq 2 \sqrt{\left|\lambda_{1} \lambda_{2}+\lambda_{2}\right|} \\
H^{4} \geq 4\left|\lambda_{1} \lambda_{2}+\lambda_{2}\right|
\end{gathered}
$$

For $V=\left\{p \in M^{2}, \lambda_{1}(p) \leq 0\right\}$ we get

$$
\int_{V} H^{4} d V \geq \int_{V} 4\left|\lambda_{1} \lambda_{2}+\lambda_{2}\right| d V
$$

If we substitute $G(p)=\lambda_{1}(p)+\lambda_{2}(p)$ in $\left|\lambda_{1}(p) \lambda_{2}(p)+\lambda_{2}(p)\right|=\left|\lambda_{2}(p) \lambda_{1}(p)+1\right|$ then we get

$$
\begin{aligned}
\left|\lambda_{1}(p) \lambda_{2}(p)+\lambda_{2}(p)\right| & =\left|\lambda_{2}(p)\left[\left(G(p)-\lambda_{2}(p)\right)+1\right]\right| \\
& =\left|\lambda_{2}(p) G(p)-\lambda_{2}^{2}(p)+\lambda_{2}(p)\right|
\end{aligned}
$$

and since $\quad \int_{V}-\lambda_{2} d V \geq-\frac{1}{2} \int_{V} G d V \quad$ in [1]

$$
\begin{gathered}
\int_{V} H^{4} d V \geq \int_{V} 4\left|\lambda_{2} G-\lambda_{2}^{2}+\lambda_{2}\right| d V \\
\geq \int_{V} 4\left|\lambda_{2}(G+1)-\lambda_{2}^{2}\right| d V
\end{gathered}
$$

since $\lambda_{2}(p)(G(p)+1)-\lambda_{2}^{2}(p) \leq 0$ on $V$

We get the inequality 


$$
\int_{V} H^{4} d V \geq \int_{V}\left(-4 \lambda_{2}(G+1)-\lambda_{2}^{2}\right) d V
$$

Since $-\frac{1}{2} G \leq-\lambda_{2}$

$$
\begin{gathered}
\int_{V} H^{4} d V \geq \int_{V}\left[-\frac{1}{2} G(G+1)-\left(-\frac{1}{2} G\right)^{2}\right] d V=\int_{V}\left(-3 G^{2}-2\right) d V \\
H^{4} \geq-3 G^{2}-2 \Rightarrow H^{2} \geq \sqrt{\left|-3 G^{2}-2\right|}
\end{gathered}
$$

Finally we get the inequality

$$
\int_{V} H^{2} d V \geq \int_{V} \sqrt{\left|-3 G^{2}-2\right|} d V
$$

\section{REFERENCES}

[1] Bang-Yen Chen, On an inequality of T.J.Willmore, Proceedings of the American Mathematical Society, 1970

[2] B.O’Neill, Semi-Riemannian Geometry, Academic Press, NewYork, 1983

Citation: S. Karagoz, "An Inequality for Closed Manifolds with Timelike Immersion and Negative Gauss Curvature ", International Journal of Scientific and Innovative Mathematical Research, vol. 6, no. 5, p. 1418, 2018., http://dx.doi.org/10.20431/2347-3142.0605002

Copyright: (c) 2018 Authors. This is an open-access article distributed under the terms of the Creative Commons Attribution License, which permits unrestricted use, distribution, and reproduction in any medium, provided the original author and source are credited. 